\title{
THE HAUSDORFF DIMENSION OF THE GRAPHS OF CONTINUOUS SELF-AFFINE FUNCTIONS
}

\author{
M. URBAŃSKI
}

(Communicated by R. Daniel Mauldin)

\begin{abstract}
The exact formula for the Hausdorff dimension of the graph of a continuous self-affine function is obtained. The Hausdorff dimension of some class of Borel probability measures is computed. The Hausdorff measures corresponding to the functions $\varphi_{c}(t)=t^{H D(\operatorname{graph}(f))} \exp (c \sqrt{\log 1 / t \log \log \log 1 / t})$ are studied.
\end{abstract}

\section{INTRODUCTION}

In the paper [K1] N. Kôno has introduced the notion of self-affine functions and in [K2] he has characterized the class of continuous self-affine functions $f:[0,1] \rightarrow[0,1]$ for which $f(0)=0$ and $f(1)=1$. The paper [K2] contains also an efficient criterion for the probability distribution of $f$ (treated as a random variable on the probability space $[0,1]$ equipped with the Lebesgue measure) to be absolutely continuous with respect to the Lebesgue measure. In [U] we have found an easy method to compute measure with respect to which the probability distribution is always absolutely continuous. As an immediate consequence of this result we have obtained Kôno's criterion. We have also shown that Kôno's condition is fulfilled if and only if the capacity (box dimension) of the graph of $f$ is equal to its Hausdorff dimension. In the present paper we improve on our study of fractal properties of the graph of $f$. Using McMullen's method (see [M]) we compute the Hausdorff dimension (see Theorem 2.4) of same class of measures on the graph of $f$ and, as the main result, we find the exact formula for the Hausdorff dimension of this graph.

Since the capacity of the graph of $f$ is almost obviously equal to $2-H$ ( $H>0$ is the parameter of $f$ described at the beginning of $\S 1$ ), Corollary 2.6 says exactly that Kôno's condition is fulfilled if and only if the capacity of the graph of $f$ is equal to its Hausdorff dimension. As we have already mentioned before, another proof of this fact has been given in [U]. In the last section of the paper we give some contribution (in our setting) to McMullen's question

Received by the editors February 7, 1989 and, in revised form, June 8, 1989.

1980 Mathematics Subject Classification (1985 Revision). Primary 28A75; Secondary 26A16.

The author was supported by the A. von Humboldt Foundation. 
about the Hausdorff measures of general Sierpiński carpets. We would like to note that our consideration in this section refers as well to this case.

\section{Preliminaries}

In this section following [K2] and [U] we collect the basic facts and definitions referring to continuous self-affine functions. We also discuss the notions of general Hausdorff measures and Hausdorff dimension.

We say that $f:[0,1] \rightarrow \mathbf{R}$ is a self-affine function of the scale parameter $H>0$ to the integer base $r \geq 4$ if and only if the following condition is satisfied:

For every $n \geq 1$, every $0 \leq k \leq r^{n}-1$ and every $0 \leq h<r^{-n}$ we have

$$
f\left(k r^{-n}+h\right)-f\left(k r^{-n}\right)=T_{k r^{-n}} \cdot r^{-H n} f\left(r^{n} h\right) \text {, }
$$

where $T_{k r^{-n}}$ equals 1 or -1 .

It is easy to see that a bounded self-affine function is continuous if and only if the above condition is fulfilled for any $0 \leq h \leq r^{-n}$. We write $I$ for $[0,1]$. From now on through the whole paper we will always assume that $f: I \rightarrow \mathbf{R}$ is a continuous self-affine function satisfying $f(0)=0, f(1)=1$ and $f(I)=$ $I$. Note that these assumptions immediately imply that $1=m r^{-H}$ where $m=\sum_{k=0}^{r-1} T_{k^{r-1}} \in\{2, \ldots, r\}$. In particular $r^{H}=m \geq 2$ is an integer and $H=\log _{r} m \leq 1$.

For any vector $V=\left[\begin{array}{l}x \\ y\end{array}\right]$ we put $|V|=x+y$. For $u=\sum_{j=1}^{\infty} u_{j} r^{-H j} \in I$ (if $u$ is $r^{H}$-adic rational we always assume that $u_{j}=0$ for $j$ large enough) let

$$
\begin{aligned}
q_{j}^{+}(u)=\operatorname{Card}\{0 \leq k \leq r-1: \text { there is } t & \in\left[k r^{-j},(k+1) r^{-j}\right) \text { such that } \\
f(t) & \left.=u \text { and } f\left(k r^{-j}\right)>f\left((k+1) r^{-j}\right)\right\} .
\end{aligned}
$$

and

$$
\begin{array}{r}
q_{j}^{-}(u)=\operatorname{Card}\left\{0 \leq k \leq r-1: \text { there is } t \in\left(k r^{-j},(k+1) r^{-j}\right]\right. \text { such that } \\
\left.f(t)=u \text { and } f\left(k r^{-j}\right)>f\left((k+1) r^{-j}\right)\right\} .
\end{array}
$$

Note that $q_{j}^{+}(u), q_{j}^{-}(u)$ depend only on $u_{1}, \ldots, u_{j}$ and denote $q_{1}^{+}(u)$ and $q_{1}^{-}(u)$ respectively by $q^{+}\left(u_{1}\right)$ and $q^{-}\left(u_{1}\right)$. For any $0 \leq k \leq r^{H}-1, u \in I$ and $j \geq 1$ we set

$$
q(k)=\left[\begin{array}{c}
q^{+}(k) \\
q^{-}(k)
\end{array}\right], \quad q_{j}(u)=\left[\begin{array}{l}
q_{j}^{+}(u) \\
q_{j}^{-}(u)
\end{array}\right], \quad Q_{k}=\left[\begin{array}{ll}
q^{+}(k) & q^{-}(\bar{k}) \\
q^{-}(k) & q^{+}(\bar{k})
\end{array}\right]
$$

and

$$
M(k)=\left|Q_{k}\left(\left[\begin{array}{c}
\frac{1}{2} \\
\frac{1}{2}
\end{array}\right]\right)\right|=\frac{1}{2}(|q(k)|+|q(\bar{k})|)=M(\bar{k}),
$$

where $\bar{k}=r^{H}-1-k$. 

that

Note that $1-u=\sum_{j=1}^{\infty} \bar{u}_{j} r^{-H j}$ and it can be easily computed (see [U, (2.9)]

By $\operatorname{Gr}(f)=\{(t, f(t)): t \in I\}$ we denote the graph of $f$.

Now let us pass to general Hausdorff measures. Let $(X, \rho)$ be a compact metric space. Let $\mathscr{B}$ be a family of subsets of $X$ such that $\bigcup \mathscr{B}=X$ and $\inf \{\operatorname{diam}(B): B \in \mathscr{B}\}=0$. Let $d: \mathscr{B} \rightarrow(0, \infty)$ be a real valued function. For $\varphi:(0, \delta) \rightarrow(0, \infty)$ and $A \subset X$ we define $H_{\varphi}(A)$, the corresponding Hausdorff measure of $A$, by

$$
H_{\varphi}(A)=\sup _{\varepsilon>0} \inf \left\{\sum_{i} \varphi\left(d\left(B_{i}\right)\right):\left\{B_{i}\right\} \subset \mathscr{B}, \bigcup_{i} B_{i} \supset A \text { and } \operatorname{diam}\left(\left\{B_{i}\right\}\right) \leq \varepsilon\right\} .
$$

In order to assure that $H_{\varphi}(A)$ is an interesting notion further restrictions on $\mathscr{B}, d$ and $\varphi$ are needed. For a discussion of this problem the reader is referred to the books [F] and [R] by Falconer and Rogers respectively. Here we mention only that usually in the literature $\mathscr{B}$ is the family of all subsets of $X$ and $d$ is the diameter function.

If $\mathscr{B}$ is the family of all subsets of $X, d$ is the diameter of sets and $\varphi(t)=t^{\alpha}$ then instead of $H_{t^{*}}(A)$ we write the shorter $H_{\alpha}(A) . H_{\alpha}(A)$ is known as the $\alpha$-dimensional Hausdorff measure of $A$. The Hausdorff dimension of $A$ is defined to be

$$
H D(A)=\inf \left\{\alpha: H_{\alpha}(A)=0\right\}=\sup \left\{\alpha: H_{\alpha}(A)=\infty\right\} .
$$

If $\mu$ is a Borel probability measure on $X$ then the Hausdorff dimension of $\mu$ is defined by

$$
H D(\mu)=\inf \{H D(Y): Y \subset X \text { and } \mu(Y)=1\}
$$

\section{PROOFS OF MAIN RESUltS}

In this section we prove the formula for Hausdorff dimension of $\operatorname{Gr}(f)$ and for some particular measures defined on $\operatorname{Gr}(f)$. For any integer $n \geq 1$ let

$$
\begin{aligned}
& T(n)=\left\{m r^{-n}: 0 \leq m \leq r^{n}-1\right\}, \\
& U(n)=\left\{m r^{-H n}: 0 \leq m \leq r^{H n}-1\right\}, \\
& G(n)=\{(t, f(t)): t \in T(n)\} .
\end{aligned}
$$

Note that $U(n)=f(T(n)), f^{-1}(U(n))=T(n)$. Let $T=\bigcup_{n=1}^{\infty} T(n), U=$ $\bigcup_{n=1}^{\infty} U(n)$ be respectively the set of $r$-adic and $r^{H}$-adic rational numbers. We also put $G=\bigcup_{n=1}^{\infty} G(n)$. For $t \in T(n), n \geq 1$ let

$$
R_{n}(t)=\left[t, t+r^{-n}\right] \times\left[f(t), f(t)+T_{t} r^{-H n}\right] .
$$

Of course $\left\{R_{n}(t)\right\}_{t \in T(n)}$ is a cover of $\operatorname{Gr}(f)$ with mutually disjoint interiors. 
Given an integer $k \geq 1$ we set $\ell=E(H k)-$ the integer part of $H k$. If $t=\sum_{n=1}^{\infty} t_{n} r^{-n} \in[0,1] \backslash T, u=\sum_{n=1}^{\infty} u_{n} r^{-H n} \in I \backslash U$, we set

$$
\begin{aligned}
S_{k}((t, u))= & {\left[\sum_{i=1}^{\ell} t_{i} r^{-i}, \sum_{i=1}^{\ell} t_{i} r^{-i}+r^{-\ell}\right] \times\left[\sum_{i=1}^{k} u_{i} r^{-H i}, \sum_{i=1}^{k} u_{i} r^{-H i}+r^{-H k}\right], } \\
\bar{S}_{k}((t, u))= & {\left[\sum_{i=1}^{\ell} t_{i} r^{-i}, \sum_{i=1}^{\ell} t_{i} r^{-i}+r^{-\ell}\right] } \\
& \times\left[\sum_{i=1}^{\ell} u_{i} r^{-H i}+\sum_{i=\ell+1}^{k} \bar{u}_{i} r^{-H i}, \sum_{i=1}^{\ell} u_{i} r^{-H i}+\sum_{i=\ell+1}^{k} \bar{u}_{i} r^{-H i}+r^{-H k}\right] .
\end{aligned}
$$

Note that if $v=\sum_{i=1}^{\ell} u_{i} r^{-H i}+\sum_{i=\ell+1}^{\infty} \bar{u}_{i} r^{-H i}$ then

$$
\bar{S}_{k}(t, u)=S_{k}(t, v) \quad \text { and } \quad \bar{S}_{k}(t, v)=S_{k}(t, u) \text {. }
$$

Let $P=\left(p_{0}, p_{1}, \ldots P_{r^{H-1}}\right.$ be a probability vector (that is, $\sum_{i=0}^{r^{H}-1} p_{i}=1$ and $\left.p_{i} \geq 0\right)$ such that $p_{i} \neq 1$ for every $i=0, \ldots, r^{H}-1$ and

$$
p_{i}=p_{\bar{i}} \text { for every } i=0, \ldots, r^{H}-1 .
$$

We shall give an easy proof of the following:

Proposition 2.1. There exists a unique probability Borel measure $\mu_{P}$ on $\operatorname{Gr}(f)$ such that for every $n \geq 1$ and every $t \in T(n)$ we have

$$
\mu_{P}(R(t))=\frac{p_{u_{1}} \cdots p_{u_{n}}}{M\left(u_{1}\right) \cdots \cdot M\left(u_{n}\right)}, \quad \text { where } \sum_{i=1}^{n} u_{i} r^{-H i}=f(t)+\frac{1}{2}\left(T_{t}-1\right) r^{-H n} \text {. }
$$

Proof. As $\left\{R_{n}(t)_{t \in T(n)}\right\}_{n=1}^{\infty}$ is an increasing sequence of partitions whose diameters tend to 0 and $\bigcap_{n=1}^{\infty} \bigcup_{t \in T(n)} R_{n}(t)=\operatorname{Gr}(f)$, there exists at most one measure that satisfies (2.2) and to prove the existence we only need to check that

$$
\sum_{j=0}^{r-1} \mu_{P}\left(R_{n+1}\left(t+j r^{-(n+1)}\right)\right)=\mu_{P}\left(R_{n}(t)\right) .
$$

Changing the way of summing we can write

$$
\sum_{j=0}^{r-1} \mu_{P}\left(R_{n+1}\left(t+j r^{-(n+1)}\right)\right)=\sum_{j=0}^{r^{H}-1} \frac{p_{u_{1}} \cdots \cdot p_{u_{n}} \cdot p_{i}}{M\left(u_{1}\right) \cdots M\left(u_{n}\right) \cdot M(i)} \cdot|q(i)| \quad \text { if } T_{t}=1
$$

and

$$
\sum_{j=0}^{r-1} \mu_{P}\left(R_{n+1}\left(t+j r^{-(n+1)}\right)\right)=\sum_{i=0}^{r^{H}-1} \frac{p_{u_{1}} \cdots p_{u_{n}} \cdot p_{i}}{M\left(u_{1}\right) \cdots \cdot M\left(u_{n}\right) \cdot M(i)}|q(\bar{i})| \quad \text { if } T_{t}=-1 .
$$


Consider the case when $T_{t}=1$. Then by (2.1) and (1.1) we get

$$
\begin{aligned}
\sum_{j=0}^{r-1} \mu_{P}\left(R\left(t+j r^{-(n+1)}\right)\right) & =\prod_{k=1}^{n} \frac{p_{u_{k}}}{M\left(u_{k}\right)} \cdot \frac{1}{2} \sum_{i=0}^{r^{H}-1} p_{i} \frac{|q(i)|+|q(\bar{i})|}{M(i)} \\
& =\prod_{k=1}^{n} \frac{P_{u_{k}}}{M\left(u_{k}\right)} \sum_{i=0}^{r^{H}-1} p_{i} \\
& =\prod_{k=1}^{n} \frac{p_{u_{k}}}{M\left(u_{k}\right)} .
\end{aligned}
$$

The case $T_{t}=-1$ we handle in exactly the same way. The proposition is proved.

Now let $\nu$ be the unique measure on $I$ determined by the condition

$$
\nu\left(\left[u, u+r^{-H n}\right]\right)=p_{u_{1}} \cdots p_{u_{n}} \quad \text { for every } n \geq 1 \text { and } u \in U(n) .
$$

Let $\pi: I \times I \rightarrow I$ denote the orthogonal projection onto the $u$-axis. In view of Proposition 2.1 we have $\pi_{*} \mu_{P}\left(\left[u, u+r^{-H n}\right]\right)=\left|q_{n}(u)\right| \cdot \prod_{k=1}^{n}\left(p_{u_{k}} / M\left(u_{k}\right)\right)$. Since $\left|q_{n}(u)\right| \leq\left|q_{n}(u)\right|+\left|q_{n}(1-u)\right|=2 \prod_{k=1}^{n} M(k)$, we obtain $\pi_{*} \mu_{P}\left(\left[u, u+r^{-H n}\right]\right) \leq$ $2 \prod_{k=1}^{n} p_{u_{k}}=2 \cdot \nu\left(\left[u, u+r^{-H n}\right]\right)$. So, we have proved the following

Lemma 2.2. The measure $\pi_{*} \mu_{P}$ is absolutely continuous with respect to $\nu$ with the Radon-Nikodym derivative bounded by 2.

If $(t, u) \in \operatorname{Gr}(f) \backslash G$ then, by (2.1), (1.1) and Proposition 2.1,

$$
\left\{\begin{array}{l}
\mu_{P}\left(S_{k}(t, u)\right)=\left|q_{k-\ell}\left(\sum_{j=1}^{\infty} u_{\ell+j} r^{-H j}\right)\right| \frac{p_{u_{1}} \cdots p_{u_{k}}}{M\left(u_{1}\right) \cdots M\left(u_{k}\right)} \\
\mu_{P}\left(\bar{S}_{k}(t, u)\right)=\left|q_{k-\ell}\left(\sum_{j=1}^{\infty} \bar{u}_{\ell+j} r^{-H j}\right)\right| \frac{p_{u_{1}} \cdots p_{u_{k}}}{M\left(u_{1}\right) \cdots M\left(u_{k}\right)}
\end{array} \quad \text { if } T_{\sum_{j=1}^{\prime} t_{j} r^{-j}}=1\right.
$$

and

$$
\left\{\begin{array}{l}
\mu_{P}\left(S_{k}(t, u)\right)=\left|q_{k-\ell}\left(\sum_{j=1}^{\infty} \bar{u}_{\ell+j} r^{-H j}\right)\right| \frac{p_{u_{1} \cdots p_{u_{k}}}}{M\left(u_{1} \cdots M\left(u_{k}\right)\right.} \\
\mu_{P}\left(\bar{S}_{k}(t, u)\right)=\left|q_{k-\ell}\left(\sum_{j=1}^{\infty} u_{\ell+j} r^{-H j}\right)\right| \frac{p_{u_{1} \cdots p_{u_{k}}}}{M\left(u_{1}\right) \cdots M\left(u_{k}\right)}
\end{array} \quad \text { if } T_{\sum_{j=1}^{\prime} t_{j} r^{-j}}=-1 .\right.
$$

In both cases we however by (2.2) obtain

$$
\begin{aligned}
& \mu_{P}\left(S_{k}(t, u)\right)+\mu_{P}\left(\bar{S}_{k}(t, u)\right) \\
& \quad=\left(\left|q_{k-\ell}\left(\sum_{j=1}^{\infty} u_{\ell+j} r^{-H j}\right)\right|_{p_{u_{1}} \cdots p_{u_{k}}}+\left|q_{k-\ell}\left(\sum_{j=1}^{\infty} \bar{u}_{\ell+j} r^{-H j}\right)\right|\right) \frac{p_{u_{1}} \cdots p_{u_{k}}}{M\left(u_{1}\right) \cdots M\left(u_{k}\right)} \\
& \quad=2 M\left(u_{\ell+1}\right) \cdots M\left(u_{k}\right) \cdot \frac{p_{u_{1}} \cdots p_{u_{k}}}{M\left(u_{1}\right) \cdots M\left(u_{k}\right)} \\
& \quad=2 \frac{M\left(u_{\ell}\right)}{M\left(u_{1}\right) \cdots M}
\end{aligned}
$$


And now we can easily prove the following:

Lemma 2.3. For $\mu_{P}$ almost every $z \in \mathrm{Gr}(f)$

$$
\lim _{k \rightarrow \infty} \frac{1}{k} \log \left(\mu_{P}\left(S_{k}(z)\right)+\mu_{P}\left(\bar{S}_{k}(z)\right)=\sum_{j=0}^{r^{H}-1} p_{j} \log \left(\frac{p_{j}}{M(j)^{H}}\right) .\right.
$$

Proof. As the functions $u=\sum_{j=1}^{\infty} u_{j} r^{-H j} \rightarrow u_{n}, n=1,2, \ldots$, are independent, identically distributed random variables with respect to the measure $\nu$, we can find by the Kolmogorov's strong law of large numbers a measurable set $E \subset I$ such that $\nu(E)=1$ and for every $u \in E$ we have

$$
\lim _{n \rightarrow \infty} \frac{1}{n} \sum_{i=1}^{n} \log p_{u_{i}}=\sum_{j=0}^{r^{H}-1} p_{j} \log p_{j}, \quad \lim _{n \rightarrow \infty} \frac{1}{n} \sum_{i=1}^{n} \log M\left(u_{i}\right)=\sum_{j=0}^{r^{H}-1} p_{j} \log M(j) .
$$

Since $\nu$ is atomless and $U$ is countable $\nu(E \backslash U)=1$. Therefore by Lemma 2.2, $\pi_{*} \mu_{P}(E \backslash U)=1$ which means that $\mu_{P}\left(\operatorname{Gr}(f) \cap \pi^{-1}(E \backslash U)\right)=1$. Now as $\operatorname{Gr}(f) \cap \pi^{-1}(I \backslash U)=\operatorname{Gr}(f) \backslash G$ and $\ell=E(H k)$, we deduce from (2.3) and (2.4) that the formula required in Lemma 2.3 holds for every $z \in(\operatorname{Gr}(f) \backslash G) \cap$ $\pi^{-1}(E)=\operatorname{Gr}(f) \cap \pi^{-1}(E \backslash U)$. The lemma is proved.

Observe now that there exists a universal integer constant $N \geq 1$ such that any subset of $I \times I$ can be covered by $N$ rectangles of the form $S_{k}(z)$ with smaller diameters. Observe also that $r^{-H k} \leq \sqrt{2} r^{-H k} \leq \operatorname{diam}\left(S_{k}(t, u)\right) \leq$ $\sqrt{r^{2}+1} r^{-H k}$. So, in order to compute the Hausdorff dimension of any subset of $I \times I$ we can use the family $\mathscr{R}=\left\{S_{k}(z)\right\}_{k \geq 1, z \in I \times I}$ and the function $e: \mathscr{R} \rightarrow(0, \infty), e\left(S_{k}(z)\right)=r^{-H k}$.

\section{Theorem 2.4.}

$$
H D\left(\mu_{P}\right)=\frac{\sum_{j=0}^{r^{H}-1}-p_{j} \log \left(\frac{p_{j}}{M(j)^{H}}\right)}{H \log r} .
$$

Proof. Let $W=\sum_{j=0}^{r^{H}-1} p_{j} \log \left(\frac{p_{j}}{M(j)}\right)$. Fix $\varepsilon>0$ and an integer $n \geq 1$. By Lemma 2.3 and Jegorov's theorem there exists a compact set $F(n) \subset \operatorname{Gr}(f)$ (which depends also on $\varepsilon$ ) and an integer $k(n, \varepsilon) \geq 1$ such that

$$
\begin{gathered}
\mu_{P}(F(n)) \geq 1-\frac{1}{n}, \\
F(n) \cap G=\varnothing, \\
W-\varepsilon \leq \frac{1}{k} \log \left(\mu_{P}\left(S_{k}(z)\right)+\mu_{P}\left(\bar{S}_{k}(z)\right)\right) \leq W+\varepsilon
\end{gathered}
$$

for every $k \geq k(n, \varepsilon)$ and $z \in F(n)$.

Take now any $k \geq k(n, \varepsilon)$ and let $\mathscr{R}_{k}=\left\{S_{k}(z): z \in F(n)\right\}, \overline{\mathscr{R}}_{k}=$ $\{\bar{S}\}_{S \in \mathscr{R}_{k}}$. Then $\bigcup \mathscr{R}_{k} \supset F(n)$ and in fact $\mathscr{R}_{k}$ consists of a finite number 
of rectangles with mutually disjoint interiors. By the definition $\overline{\mathscr{R}}_{k}$ consists of the same number of rectangles which by (2.0) have also mutually disjoint interiors. Hence by (2.7)

$$
\begin{aligned}
\sum_{s \in \mathscr{R}_{k}} e(S)^{(-W+\varepsilon) / H \log r} & =\sum_{S \in \mathscr{R}_{k}}\left(r^{-k H}\right)^{(-W+\varepsilon) / H \log r} \leq \sum_{S \in \mathscr{R}_{k}}\left(\mu_{P}(S)+\mu_{P}(\bar{S})\right) \\
& =\mu_{P}\left(\bigcup \mathscr{R}_{k}\right)+\mu_{P}\left(\bigcup \overline{\mathscr{R}}_{k}\right) \leq 2 .
\end{aligned}
$$

So, letting $k \rightarrow \infty$ we get $H_{(-W+\varepsilon) / H \log r}(F(n)) \leq 2$ and therefore $H D(F(n)) \leq$ $(-W+\varepsilon) / H \log r$. Hence $\mu_{p}\left(\bigcup_{n=1}^{\infty} F(n)\right)=1$ and $H D\left(\bigcup_{n=1}^{\infty} F(n)\right) \leq(-W+\varepsilon) /$ $H \log r$. Letting $\varepsilon \backslash 0$ we therefore get $H D\left(\mu_{p}\right) \leq-W / H \log r$.

Now let $X \subset \operatorname{Gr}(f)$ be any Borel subset of $\operatorname{Gr}(f)$ such that $\mu_{P}(X)=1$. Take $k \geq k(2, \varepsilon) \quad(\varepsilon$ is again fixed) and consider $\mathscr{M} \subset \mathscr{R}$ a cover of $X$ such that $e(S) \leq r^{-H k}$ for every $S \in \mathscr{M}$. Let $\mathscr{N}=\{S \in \mathscr{M}: S \cap F(2) \neq \varnothing\}$. Then by (2.6) $\bigcup \mathscr{N} \supset F(2) \cap X$ and by (2.7)

$$
\begin{aligned}
\sum_{S \in \mathscr{M}} e(S)^{(-W-\varepsilon) / H \log r} & \geq \sum_{S \in \mathscr{N}} e(S)^{(-W-\varepsilon) / H \log r} \\
& \geq \sum_{S \in \mathscr{N}}\left(\mu_{P}(S)+\mu_{P}(\bar{S})\right) \\
& \geq \sum_{S \in \mathscr{N}} \mu_{P}(S) \geq \mu_{P}(\bigcup \mathcal{N}) \\
& \geq \mu_{P}(X \cap F(2)) \\
& =\mu_{P}(F(2)) \\
& \geq \frac{1}{2} .
\end{aligned}
$$

So letting $k \rightarrow \infty$ and next $\varepsilon \rightarrow 0$ we obtain $H D(X) \geq-W / H \log r$. Hence $H D\left(\mu_{P}\right) \geq-W / H \log r$. The proof is finished.

Theorem 2.5.

Proof. Let

$$
H D(\operatorname{Gr}(f))=\frac{\log \left(\sum_{j=0}^{r^{H}-1} M(j)^{H}\right)}{H \log r} .
$$

$$
\delta=\frac{\log \left(\sum_{j=0}^{r^{H}-1} M(j)^{H}\right)}{H \log r} .
$$

Then $r^{\delta H}=\sum_{j=0}^{r^{H}-1} M(j)^{H}$ and thus putting $\alpha_{j}=M(j)^{H} r^{-\delta H}, j=0,1, \ldots$, $r^{H}-1$, we define a probability vector $\alpha=\left(\alpha_{1}, \ldots, \alpha_{n}\right)$. Suppose that $\alpha_{i}=1$ for some $i=0, \ldots, r^{H}-1$. Then $M(i)=r^{\delta}$ and by the definition of $\delta$, $\log \left(M(i)^{H}\right)=\log \left(\sum_{j=0}^{r^{H}-1} M(j)^{H}\right)$. As $M(j) \geq 1$ for every $j=0, \ldots, r^{H}-1$ 
we therefore conclude that $r^{H}-1=0$. This is a contradiction as $H>0$. So, we can apply Theorem 2.4 and we get

$$
H D(\operatorname{Gr}(f)) \geq H D\left(\mu_{\alpha}\right)=\frac{\sum_{j=0}^{r^{H}-1} \alpha_{j} \log \left(r^{-\delta H}\right)}{H \log r}=\delta .
$$

So, we only need to prove that $H D(\operatorname{Gr}(f)) \leq \delta$. By (2.3) and the definition of $\alpha$, for every $z=(t, u) \in \operatorname{Gr}(f) \backslash G$ and $k \geq 1$ we have

$$
\mu_{\alpha}\left(S_{k}(t, u)\right)+\mu_{\alpha}\left(\bar{S}_{k}(t, u)\right)=2 \frac{M\left(u_{1}\right)^{H} \cdots M\left(u_{k}\right)^{H}}{M\left(u_{1}\right) \cdots M\left(u_{\ell}\right)} r^{-\delta H k} .
$$

Since $\ell=E(H k)$, it can be easily shown (for the exact proof see [M, Lemma 4a] that

$$
\limsup _{k \rightarrow \infty}\left(\frac{M\left(u_{1}\right)^{H} \cdots M\left(u_{k}\right)^{H}}{M\left(u_{1}\right) \cdots M\left(u_{\ell}\right)}\right)^{1 / k} \geq 1 .
$$

Fix $\varepsilon>0$, an integer $n \geq 1$ and define the sequence $\left\{\mathscr{K}_{k}\right\}_{k=n}^{\infty}$ of subfamilies of $\mathscr{R}$ setting

$$
\mathscr{K}_{k}=\left\{S_{k}(t, u): \frac{M\left(u_{1}\right)^{H} \cdots \cdot M\left(u_{k}\right)^{H}}{M\left(u_{1}\right) \cdots \cdot M\left(u_{\ell}\right)} \geq r^{-\varepsilon H k},(t, u) \in \operatorname{Gr}(f) \backslash G \backslash \bigcup_{j=n}^{k-1} \bigcup \mathscr{K}_{j}\right\}
$$

So $\mathscr{R}^{(n)}=\bigcup_{k=n}^{\infty} \mathscr{K}_{k} \subset R$ consists of rectangles with mutually disjoint interiors. By (2.0) the same refers to the family $\overline{\mathscr{R}}^{(n)}=\{\bar{S}\}_{S \in \mathscr{R}^{(n)}}$. Therefore by (2.9) $\sum_{S \in \mathscr{R}^{(n)}} e(S)^{\delta+\varepsilon} \leq \sum_{S \in \mathscr{R}^{(n)}}\left(\mu_{\alpha}(S)+\mu_{\alpha}(\bar{S})\right)=\mu_{\alpha}\left(\bigcup \mathscr{R}^{(n)}\right)+\mu_{\alpha}\left(\bigcup_{\mathscr{R}^{(n)}} \leq 1+\right.$ $1=2$. As by $(2.10)$ we have $\bigcup \mathscr{R}^{(k)} \supset \operatorname{Gr}(f) \backslash G$, letting $n \rightarrow \infty$ we get $H_{\delta+\varepsilon}(\operatorname{Gr}(f) \backslash F) \leq 2$. Hence $H D(\operatorname{Gr}(f) \backslash G) \leq \delta+\varepsilon$ for every $\varepsilon>0$. Thus $H D(\operatorname{Gr}(f) \backslash G) \leq \delta$ and since $G$ is countable $H D(\operatorname{Gr}(f)) \leq \delta$. The theorem is proved.

As an immediate consequence of this theorem we get the following result proved in [U, Theorem 4.2 and Theorem 4.3].

Corollary 2.6. $H D(\operatorname{Gr}(f))=2-H$ if and only if $|q(j)|+|q(\bar{j})|=2 r^{1-H}$ for every $j=0,1, \ldots, r^{H}-1$.

\section{Additional RESUlts}

We begin this section with the following

Lemma 3.1. If $\left\{X_{n}\right\}_{n=1}^{\infty}$ is a sequence of uniformly bounded identically distributed independent random variables with positive variance $\sigma^{2}$, then for every $0 \leq a \leq 1$ we have almost surely

$$
\limsup _{n \rightarrow \infty} \frac{a S_{n}-S_{E(a n)}}{\sqrt{2 \sigma^{2} n \log \log n}}=\sqrt{a(1-a)}, \quad \text { where } S_{n}=\sum_{k=1}^{n} X_{k} .
$$


Proof. Let $K=\left\{f \in C(I): f(x)=\int_{0}^{x} g(t) d t\right.$ for every $x \in I$ where $\left.\int_{0}^{1} g^{2}(t) d t \leq 1\right\}$ and for every $n \geq 1$ let $f_{n}: I \rightarrow \mathbf{R}$ be the linear interpolation of the set $\left\{S_{i} / \sqrt{2 \sigma^{2} n \log \log n}\right\}_{i=0}^{n-1}$. It is proved in [S] that the set of accumulation points of $\left\{f_{n}\right\}_{n=1}^{\infty}$ (with the supremum-normed topology on $C(I)$ ) coincides almost surely with $K$.

So, in order to compute the upper limit required in the lemma it is sufficient to find the supremum of the functional $F: K \rightarrow \mathbf{R}$ given by $F(f)=a f(1)-f(a)$. But as $f(x)=\int_{0}^{x} g(t) d t$ where $\int_{0}^{1} g^{2}(t) d t \leq 1$, we have

$$
F(f)=a \int_{0}^{1} g(t) d t-\int_{0}^{a} g(t) d t=(a-1) \int_{0}^{a} g(t) d t+a \int_{a}^{1} g(t) d t
$$

Now it is easy to see that this supremum is attained on the functions $g$ which are constant on the intervals $[0, a)$ and $[a, 1]$. Therefore we only need to find the supremum of the function $h: \mathbf{R}^{2} \rightarrow \mathbf{R}$ defined by

$$
h(x, y)=(a-1) a x+a(1-a) y, \quad \text { where } a x^{2}+(1-a) y^{2} \leq 1
$$

Since $a(1-a)>0$ this supremum is attained on a pair $(x, y)$ where $x \leq 0$, $y \geq 0$ and $a x^{2}+(1-a) y^{2}=1$. So, we have come to an ordinary extremum problem in calculus of one variable and its solution gives that our upper limit is equal to $\sqrt{a(1-a)}$.

Now suppose that $M(j) \neq r^{1-H}$ for at least one $j=0, \ldots, r^{H}-1$. Taking on $X_{k}=\log M\left(u_{k}\right)$ and $a=H$ we deduce from Lemma 3.1, Lemma 2.2 and (2.9) the following:

$$
\limsup _{k \rightarrow \infty} \frac{\log \left(\mu_{\alpha}\left(S_{k}(t, u)\right)+\mu_{\alpha}\left(\bar{S}_{k}(t, u)\right)\right)+H \delta k \log r}{\sqrt{2 \sigma^{2} k \log \log k}}=\sqrt{H(1-H)}
$$

for $\mu_{\alpha}$ - a.e. $(t, u) \in \operatorname{Gr}(f)$ where $\sigma^{2}=\int\left(\log M\left(u_{1}\right)-\int \log M\left(u_{1}\right) d \nu_{\alpha}\right)^{2} d \nu_{\alpha}>$ 0 . Now for any $c>0$ let $\varphi_{c}(t)=t^{\delta} \exp (c \sqrt{\log 1 / t \log \log \log 1 / t})$. Proceeding analogously as in the proof of Theorem 2.4 (the role of Lemma 2.3 is played now by formula (3.1)) we can prove the following.

Theorem 3.2. (a) The measure $\mu_{\alpha}$ is absolutely continuous with respect to $H_{\varphi}$ for every $c>\sqrt{2 \sigma^{2}(1-H) / \log r}$.

(b) The measure $\mu_{\alpha}$ is singular with respect to $H_{\varphi_{c}}$ for every $c<$ $\sqrt{2 \sigma^{2}(1-H) / \log r}$. 
Taking $c=0$ we get immediately the following:

Corollary 3.3. The measure $\mu_{\alpha}$ is singular with respect to $H_{\delta}$.

This exactly says that we can find a Borel set $X \subset \operatorname{Gr}(f)$ such that $\mu_{\alpha}(X)=1$ and $H_{\delta}(X)=0$ and therefore it can be treated as a contribution to McMullen's question (posed in $[\mathrm{M}]$ in the context of general Sierpinski carpets) of whether $H_{\delta}\left(q_{r}(t)\right) \neq 0$. It suggests rather that one should expect $H_{\delta}(\operatorname{Gr}(f))=0$ (unless $M(j)=r^{1-H}$ for every $\left.j=0,1, \ldots, r^{H}-1\right)$. However the problem is still open.

\section{ACKNOWLEDGMENT}

The author wishes to thank A. Jakubowski for showing him a method of proving Lemma 3.1 .

\section{REFERENCES}

[F] K. J. Falconer, The geometry of fractal sets, Cambridge University Press, 1985.

[K1] N. Kônc, On self-affine functions, Japan J. Appl. Math. 3 (1986), 271-280.

[K2] __ On self-affine functions II, Japan J. Appl. Math. 5 (1988), 441-454.

[M] C. McMullen, The Hausdorff dimension of general Sierpinski carpets, Nagoya Math. J. 96 (1984), 1-9.

[R] C. A. Rogers, Hausdorff measures, Cambridge University Press, 1970.

[S] V. Strassen, An invariant principle for the law of the iterated logarithm, Z. Wahrscheinlichkeitstheorie 3 (1964), 211-226.

[U] M. Urbański, The probability distribution and Hausdorff dimension of self-affine functions, (to appear in Prob. Th. and Rel. Fields).

Instytut Matematyki, Uniwersytet M. Kopernika, ul. Chopina 12/18, 87-100 Toruń, Poland 